\title{
La frontera Chiapas-Guatemala como objeto de estudio de la antropología. Años ochenta del siglo $\mathrm{XX}$ \\ The Chiapas-Guatemala Border as an Object of Anthropological Study: The Eighties in the Twentieth Century
}

\author{
Carlos Gutiérrez Alfonzo \\ Centro de Investigaciones Multidisciplinarias sobre Chiapas y la Frontera Sur-UNAM \\ galfonzo@unam.mx
}

ORCID:

Resumen:

Indago aquí sobre cuáles acepciones de frontera fueron preponderantes en textos de científicos sociales y qué se analizó mediante estas designaciones en la frontera Chiapas-Guatemala, estudiada a partir de los años ochenta del siglo XX, cuando surgió como campo de investigación de la antropología. Seleccioné un número de textos de los años iniciales, con el objetivo de conocer esos primeros planteamientos teórico-metodológicos con los que se exploró esta zona. La línea adoptada me permitió verificar que los investigadores se tomaron libertades al momento de definir los términos teóricos de sus exploraciones. Fueron elegidas dos regiones de estudio (el Soconusco y la Selva Lacandona, que colindan con los departamentos guatemaltecos de San Marcos y Petén), las cuales se iban a ir convirtiendo en polos de atracción para los interesados en conocer esta frontera.

Palabras clave: frontera, Estado-nación, antropología, Chiapas, Guatemala.

Abstract:

This research note looks into the definitions of the notion of border prevailing in social science texts. These definitions were analyzed in relation to the Chiapas-Guatemala border, which it studies since the 1980s, when it emerged as an area of research in the field of anthropology. A number of texts from the initial years were selected in order to identify the first theoretical-methodological formulations with which this region was studied. This line of research enabled the confirmation that the researchers in fact took liberties at the moment of defining the theoretic terms of their research. Two regions of study were selected: Soconusco and the Lacandón rainforest, which border the Guatemalan counties of San Marcos and Petén. These counties were gradually becoming points of attraction for those who were interested in getting to know that border.

Keywords: border, nation-State, anthropology, Chiapas, Guatemala. 


\section{Introducción}

E n las ciencias sociales, en especial en la antropología, la frontera sur ${ }^{1}$ se reconoció como objeto de estudio en la década de los ochenta del siglo XX. Antes, el sureste mexicano era la referencia (De Vos 2002:49). Hubo dos iniciativas académicas con las cuales se examinó la existencia de esta franja territorial. La primera fue el proyecto sobre La formación histórica de la frontera sur, impulsado por el Centro de Investigaciones y Estudios Superiores en Antropología Social, y dirigido por Andrés Fábregas Puig, de 1983 a 1985; la segunda, el foro denominado "El redescubrimiento de la frontera sur", cuya sede fue la ciudad de Mérida, Yucatán, en octubre de 1986 (De Vos 2002:49).

Hace 32 años fue publicado el libro La formación histórica de la frontera sur, en 1985, producto de la investigación coordinada por Fábregas Puig. El foro de Mérida tuvo como resultado un libro que llevó el mismo título, El redescubrimiento de la frontera sur. Apareció en 1989. Fue compilado por Jesús Hernández Palacios y Juan Manuel Valenzuela. He hecho un recuento de la bibliografía surgida a partir de entonces. Conforme avanzaba en esta labor, se me fue imponiendo como interrogante el concepto de frontera. En este artículo me he propuesto observar si se partió de un concepto de frontera y si con base en este fue definido el universo que se pretendía conocer. Me circunscribo a los resultados expuestos en las primeras investigaciones antropológicas. Voy a ir analizando los textos en busca del que ha sido mi propósito, como lo he explicado líneas arriba. He divi-

1 En la ciudad de Puebla, las comisiones científicas de México y Guatemala firmaron los documentos en que se marcaba la línea divisoria entre ambos países; rubricarlos fue el signo de que se había llegado a la definición de la frontera. Esto ocurrió en 1899 (Caballero, 2014:37), 17 años después de haberse suscrito el Tratado de Límites. Hubo tensiones generadas por el diferendo con respecto a la definición de la línea. Uno y otro país mostraron sus desavenencias. Estas posiciones impidieron por muchos años que se llegara a acuerdos en relación con las temáticas fronterizas. Los conflictos en Centroamérica, en las décadas de los setenta y los ochenta del siglo XX, obligaron a que la frontera fuera vista por las repercusiones que podían tener los movimientos sociales centroamericanos en el sur de México (Fábregas, 1985, Castillo et. al., 2006: 266). Fue el tiempo del descubrimiento de yacimientos petroleros, de la construcción de plantas hidroeléctricas y del establecimiento de un polo de desarrollo turístico (Fábregas, 2011: 77). Las migraciones de centroamericanos y de chiapanecos en busca del norte, la intención de establecer, en un momento, acuerdos de integración económica que abarcaran la región centroamericana, el alzamiento armado en Chiapas, las iniciativas en pos de un manejo sostenible de los recursos naturales de la región y los planes de seguridad nacional, más allá de los límites territoriales, provocaron que se observara la frontera sur con base en los desafíos que ha impuesto "la geopolítica contemporánea" (Castillo et al., 2006: 266). Las expuestas, a grandes rasgos, son etapas políticas de la frontera sur (Castillo et al., 2006: 266). Un acercamiento microscópico dejará ver las relaciones entre habitantes de ambos lados de la línea. Ubicable debe ser también la idea de un continuum cultural aún ostentada, sobre todo, en círculos académicos. 
dido el artículo en cinco partes. En la primera observo cómo se comprendió la frontera en tanto región; en la segunda, a partir de transformaciones religiosas; en la tercera, con base en la condición étnica; de acuerdo con la cultura y la política, en la cuarta. La quinta es una síntesis de lo expuesto.

\section{Región y frontera}

Una referencia obligada en esta indagación con respecto a la frontera ChiapasGuatemala fue la que descubrí en el estudio antropológico promovido por el CIESAS. Andrés Fábregas Puig ha contado cómo a mediados de los años ochenta del siglo XX el entonces director de dicho Centro le expuso que asumiera el estudio de la frontera sur mexicana (Fábregas, 2011: 17). Se trató de una iniciativa institucional. Los términos académicos fueron definidos en la coordinación de proyectos especiales del CIESAS. Una vez aceptado por su director, a quien le fue presentado el proyecto, el financiamiento provino del Programa Cultural de las Fronteras. Por ese medio se impulsó que los directores o secretarios de los organismos estatales de cultura colaboraran con los investigadores del proyecto (Fábregas, 1985: 1). Hubo acción política por parte de Andrés Fábregas, coordinador de la investigación, tanto hacia los estados como hacia la administración federal. El quehacer antropológico comandado por una institución académica mexicana adquirió realce en la frontera sur.

El 10 de junio de 1985, en Tuxtla Gutiérrez, Andrés Fábregas expuso lo siguiente:

Es importante manifestar que el grupo de investigación de la Frontera Sur, constituido por profesionales que egresaron de diferentes centros educativos del país, tuvo una disciplina y un desempeño dignos de encomio. Sobre todo, retomaron la tradición de estar en el campo_en estos tiempos en que los antropólogos mexicanos son cada día más renuentes a abandonar al [sic] D. F. y sus zonas aledañas. El trabajo desempeñado por el grupo de investigación ha constituido el cimiento que permitió la fundación del CIESAS-Sureste (Fábregas, 1985: 2).

La frontera sur se abrió como la gran posibilidad para que un grupo de investigadores estuviera en el campo y se hiciera antropología. Al limitar el proyecto "La formación histórica de la frontera sur", Fábregas tuvo como guía las investigaciones de Manuel Gamio, Gonzalo Aguirre Beltrán y Ángel Palerm. Ellos tres pusieron el énfasis en la región. Y para adentrarse en la historia particular, Pueblo 
en vilo, de Luis González y González, le resultó una fuente confiable. La ventaja, precisó Fábregas, "del análisis de la historia local — que es el problema de la región- está en que su dimensión concreta presenta la riqueza y complejidad de la práctica social que constituye la historia nacional" (Fábregas, 1985: 6). La práctica social se define en términos históricos y culturales, y engloba "la cultura, la economía, la ideología, la política y la sociedad dentro de historias particulares que son el sustento de la vida nacional" (Fábregas, 1985: 6).

La regionalización propuesta en el proyecto tuvo como punto de partida "la sociedad colonial" (Fábregas, 1985: 6). Es en esa época en que la región empieza a asumir las características que le habrán de ser propias. Fueron surgiendo grupos políticos locales, con relaciones disímiles o empáticas, a veces, con los poderes centrales. Fueron estos grupos los que se movilizaron para decidir los destinos del área: "en el contexto de América Latina en general y México en particular, el Estado nacional es un producto que resulta de las contradicciones introducidas por el colonialismo" (Fábregas, 1985: 7).

Esa perspectiva amplia, tanto para observar las etapas históricas como para percibir las relaciones con los poderes centrales, le hace decir a Andrés Fábregas que "los elementos de definición de una región tienen que tomarse de la historia compartida, de las relaciones mediadas, entre la historia general y la historia concreta en un proceso en el que se van entrelazando las tradiciones locales para resultar en la historia nacional" (Fábregas, 1985: 7).

La región vista por medio de historias diversas, en concordancia con el Estado nacional. Esa fue la propuesta metodológica del proyecto. Apareció la frontera como producto de relaciones entre poderes locales y poderes de alcance nacional. La definición del espacio de estudio se basó en el apelativo existente hasta entonces: "sureste mexicano". Se reconoció su complejidad, producto de su extensión. La diversidad en esta gran área había que decirla: "En el sureste habitan mayoritariamente los descendientes de los pueblos originales. No es exagerado afirmar que en este amplio territorio vive la gran mayoría del México indio, con todo su vigor cultural y sus añejos problemas" (Fábregas, 1985: 8). Otro rasgo a considerar fue el siguiente: "la comunidad como unidad corporada pero nunca aislada o encerrada en sí misma" (Fábregas, 1985: 10).

La frontera sur fue comprendida de esta forma: "No puede hablarse con propiedad de la frontera sur como una sóla [sic] región sino más bien como un territorio en donde coexisten diferentes ámbitos regionales que son el resultado de procesos sociales iniciados hace cuatrocientos años [...]. México como nación 
comparte una historia con los pueblos de Centroamérica y más allá de las diferencias particulares, conforma un continum cultural junto con el resto de América Latina" (Fábregas, 1985: 16). Dos cuestiones en juego en estas ideas expuestas: la nacionalidad y el vínculo con Centroamérica, con América Latina. Y la frontera sur con su importancia en estos dos puntos.

La noción de frontera estuvo circunscrita al espacio geográfico, delimitado por intereses nacionales. Y en esta área se realizó el proyecto de referencia. Uno de los objetivos fue conocer cómo se había formado esta parte del país. La investigación se hizo en los cinco estados de la frontera sur, con base en determinadas temáticas, según lo explicó Fábregas (1985: 17-22). Al coordinador del proyecto le interesaba pensar la nación a partir de una región con una frontera política.

En la introducción del libro La formación histórica de la frontera sur (1985), que he estado citando, el autor puntualizó que la institución financiadora del proyecto respetó las decisiones académicas del equipo de investigación. A su vez, él dejó en manos de los investigadores el esclarecimiento de la forma en que había de alcanzarse cada objetivo propuesto. Me interesa citar dos textos que fueron resultado de este proyecto. El de Juan Pohlenz, "La conformación de la frontera entre México y Guatemala. El caso de Nuevo Huixtán en la selva Chiapaneca" (1985), y el de Patricia Ponce, Palabra viva del Soconusco: nuestra frontera sur (1985). Sostengo esta elección por dos motivos que están relacionados con lo que indago al escribir este artículo: la cuestión metodológica y la centralidad de la investigación en regiones específicas.

Juan Pohlenz elaboró una escala de análisis. La definió por medio de relaciones. Su intención era esclarecer la formación de la frontera sur:

a) el nivel de la política internacional, que incluye, desde la fijación de los límites en el siglo pasado, las medidas de política que afectan a la región tomadas por el Estado mexicano a partir de intereses nacionales y en función de las correlaciones multi y bilaterales; b) el nivel de la formación regional, en el que se sintetiza la acción de todos los niveles de la realidad; y c) el nivel de la interacción social entre la población fronteriza asentada a ambos lados de la línea (Pohlenz, 1985: 25).

Alcanzó detalles que, en la propuesta de Fábregas, citada líneas arriba, están diluidos por la generalidad de su aserto. Se trata de niveles sugerentes. Pohlenz justificó que dedicaría su quehacer a la formación regional. Estudiará la frontera en la fracción correspondiente al estado de Chiapas y expondrá un estudio de caso. Sus investigaciones anteriores lo habían colocado en ciertas áreas de su 
elección. Estableció que la región cultural de la frontera sur era la de los Altos de Chiapas y Guatemala. Recurrió a una continuidad ofrecida, sobre todo en la arqueología, por la presencia de población hablante de alguna lengua catalogada como "maya".

Observará regiones de la frontera Chiapas-Guatemala. Pondrá el énfasis en la del Soconusco y en la de la Selva. Entreverá la de la Sierra Madre y la de Los Llanos (Pohlenz, 1985: 55). Dirá que tres fases son distinguibles, después de la formación de los Estados nacionales de México y Guatemala: "Implantación del capitalismo, consolidación del Estado Burocrático Autoritario y monopolismo de Estado" (Pohlenz, 1985: 55). Puntualizará que la denominación "Frontera sur" será nueva, en el momento en que se hizo la investigación. Al hablar de frontera siempre se tenía en mente la del norte de México (Pohlenz, 1985: 25). Privilegió los datos históricos. Y el caso de estudio apareció como algo aún sin concluir. Las escalas fueron un aporte; su dimensión sobrepasó las características y los tiempos de la investigación, como él mismo lo advirtió (Pohlenz, 1985).

Patricia Ponce Jiménez, para presentar historias de vida de personas habitantes del Soconusco, tuvo como referencia que la frontera sur fue establecida por una razón de Estado. A esa decisión se imponía "una gran línea de contacto y continuidad cultural" (Ponce, 1985: 14), que abarcaba Centroamérica y el Caribe. Observó una región, la del Soconusco, compleja en términos culturales, con vitalidad en la época prehispánica por ser la ruta que conectaba el centro de México con Centroamérica. La cultura regional distaba mucho de ser una adaptación de la "nacionalidad" mexicana, más bien, se fue imponiendo el capital como signo del Estado-nación (Ponce, 1985: 15).

Ponce Jiménez encontró la vida fronteriza expresada en la condición de los trabajadores: "la nacionalidad, antes que una dimensión ideológica es un uso económico-social para explotar al máximo la fuerza de trabajo en su conjunto" (Ponce, 1985: 15). Su apreciación se sostuvo en que el trabajador mexicano estaba sujeto a la "competencia desleal" originada por el trabajador centroamericano, "que vende su fuerza de trabajo muy por debajo de su valor y sin ninguna prestación social" (Ponce, 1985: 15).

Los vínculos con Guatemala, en términos culturales, las relaciones de parentesco y las migraciones laborales de guatemaltecos hacia el Soconusco le hicieron decir a esta autora que "el Soconusco vive, desde antes de su integración política a México, un proceso de 'centroamericanización' en detrimento del proyecto 
de unidad nacional que persigue el gobierno central mexicano, como proyecto de consolidación" (Ponce, 1985: 16).

Vio que una de las características del Estado mexicano era la centralización del poder, cuya concentración estaba bajo el influjo de condicionantes: la región fronteriza y su proceso histórico, la imposición del capital y los grupos de poder locales. "Esto da como resultado un proceso contradictorio de identificación como mexicanos ante el país vecino (Guatemala), pero al mismo tiempo un desarraigo respecto a la relación entre la región y el centro del país" (Ponce, 1985: 19).

\section{Fronteras a partir del cambio religioso}

El enfoque de la frontera como región adquirió otro sesgo cuando en el CIESASSureste se puso el énfasis en el estudio de la conversión religiosa que se estaba viviendo en los estados del sureste mexicano. Esa temática fue impulsada en el segundo lustro de los ochenta del siglo XX y la coordinación corrió a cargo de Fábregas. Se trató de un proyecto colectivo. El sustento teórico se buscó en los estudios antropológicos sobre religión, en ese vínculo entre capitalismo y ética protestante investigado por Max Weber, en la tesis de la religión como sistema cultural expresada por Clifford Geertz y en la de la religión como sistema de identidad: la existencia de rasgos de identidad, entre ellos el de la religión, junto con los de grupo, clase y nación (Fábregas, 1989: 13). Y al situar la religión en el ámbito de las relaciones de producción, los planteamientos de Karl Marx resultaron fundamentales. Se era consciente de que había que partir de lo que se deseaba conocer:

Es necesario revisar a los clásicos (como Durkheim, Marx o Weber) pero con los ojos puestos en la realidad actual. Es indudable que existe una correlación entre religión y estructura social, pero no sucede diariamente como quiso Durkheim, sino que está establecida a través de mediaciones concretas importantes de identificar en sus contextos correspondientes (Fábregas, 1989: 17-18).

El cambio religioso analizado como parte de las relaciones sociales, en el contexto que ofrecía en ese momento el sureste de México. Al planteamiento teórico se le agregó un procedimiento metodológico, expresado por Carlos Guzmán Böckler: 
Cuando nos dispusimos a recolectar la información, partimos de la proposición de Guillermo Bonfil que reducida a muy pocas palabras se puede anunciar así: que sean los actores sociales investigados, tanto colectiva como individualmente, quienes se expresen, correspondiéndonos a nosotros recoger sus manifestaciones tal como sean expresadas, para luego sistematizarlas y, en la medida de lo posible, interpretarlas respetando sus diferencias y similitudes, así como su espontaneidad (Guzmán, 1989: 29).

Fueron elegidas dos localidades ubicadas en la frontera Chiapas-Guatemala: Tziscao y Cuauhtémoc, en el municipio de La Trinitaria. Las investigaciones en esos lugares estuvieron bajo la responsabilidad de Jorge Luis Cruz Burguete y Rosalba Aída Hernández Castillo. El primero justificó de esta forma la selección de la localidad:

Después de realizar un recorrido por la región Fronteriza y parte de la Selva, decidí concentrar mis observaciones sobre la comunidad de Tziscao por ser esta la más antigua de la región, encontrarse a escasos metros de la línea divisoria, contar con refugiados de diversas aldeas guatemaltecas y, lo más importante, por la presencia de siete grupos religiosos distintos al interior de dicha localidad (Cruz, 1989: 40).

A la noción de frontera como espacio político, Cruz agregó las de etnia y clase social; estaba en consonancia con los planteamientos teóricos definidos en el proyecto. La frontera sur chiapaneca era el territorio para investigar esos preceptos a la luz de las características regionales, en relación con el mercado nacional, y de las especificidades de los movimientos populares, sociales y religiosos (Cruz, 1989: 39). El investigador comprendía que el hecho social estaba en el centro de la discusión, lo que implicaba "no extralimitar las explicaciones que sólo corresponden a los fenómenos regionales. [...] El compromiso consiste en dar cuenta de los hechos y cambios sociales en su justa dimensión, temporal, espacial" (Cruz, 1989: 64).

La etnia, la clase social y la frontera estuvieron dentro de las preocupaciones del investigador. Los habitantes de la localidad estudiada respondieron a los cuestionamientos que él hizo en relación con la práctica religiosa. El investigador describió cómo la población se agrupaba de acuerdo con el credo profesado. Cómo entraban en conflicto uno y otro grupo. En el recuento histórico mostró cómo durante la primera mitad del siglo XX los pueblos de uno y otro lado de la frontera mantuvieron contacto: "El parentesco, el comercio, el deporte y los festejos religiosos eran motivos suficientes para esa estrecha relación" (Cruz, 1989: 
45). Centrado en la temática que lo llevó a la localidad, identificó que con los refugiados llegaron al ejido algunos credos no católicos y que la Teología de la Liberación se asentó a lo largo de las regiones Selva y Fronteriza para atender a la población refugiada. La Teología vio hacia los refugiados, hacia Guatemala. Los refugiados, una vez ubicados en el ejido, se movieron hacia "las sectas".

El investigador se movió dentro de un tiempo y un espacio amplios que le permitieran comprender lo que observara. Tuvo como asideros históricos los movimientos sociales en Centroamérica, los modos de producción capitalista y el imperialismo en busca de su propio beneficio (Cruz, 1989: 108). En un nivel cercano a su objeto de estudio, ubicó la formación de la frontera como parte del proyecto del Estado mexicano. Al establecerse la frontera, se dio lugar a la figura del transmigrante: alguien que iba y venía de uno y otro lado, sometido a esta nueva condición. La frontera de los años ochenta del siglo XX viró. Había sido un espacio cultural, de intercambio, de vínculos sociales. Se convirtió en un espacio en el que de un lado estaban las masacres; y del otro, la posibilidad de salvar la vida (Cruz, 1989: 108). Hay en el investigador un afán de colocar la religión cosmogónica como el sustrato de la relación del hombre con el cosmos. La da como un halo que cubriera esa zona de la frontera México-Guatemala. Ubicable en el rezo a la cruz, en una que otra ceremonia. Sitúa por igual la Teología de la Liberación; es la que impulsó el movimiento social en esta zona del país.

Rosalva Aída Hernández Castillo se situó en el ejido Cuauhtémoc, del municipio de La Trinitaria, que está a siete kilómetros al oriente de Tziscao, sobre la línea fronteriza Chiapas-Guatemala: "un pequeño ejido cafetalero (1519 personas) (que) presenta una diversidad cultural al ser resultado de la unión de dos grupos lingüísticos; una diversidad socio-política al reunir a ejidatarios mexicanos y refugiados guatemaltecos y diversidad religiosa, al existir cinco grupos religiosos diferentes" (Hernández, 1989: 125). Elogio de lo diverso como justificación del lugar elegido para hacer la investigación sobre cambio religioso. Una localidad ubicada en la frontera México-Guatemala, dos expresiones lingüísticas, ejidatarios y refugiados y grupos religiosos. Y el compromiso por parte de la investigadora de escribir mediante documentos y testimonios orales la historia subvertida de esta población al trazarse los límites fronterizos.

Las expresiones de la costumbre fueron para la investigadora el sustrato de su objeto de estudio. De ahí partió para observar los cambios en las expresiones re-

2 Carolina Rivera Farfán me ha explicado, mediante comunicación personal, que el investigador utilizó esta definición (Cruz, 1989: 60-61), que se ha vuelto peyorativa; agregó que en los estudios de las religiones y la religiosidad hace tiempo que se vio su inviabilidad. 
ligiosas asumidas por los habitantes del ejido. Visitó el lugar durante un año, con estancias de hasta un mes. Llevó a cabo observación participante y entrevistas estructuradas y no estructuradas (Hernández, 1989: 128). La localidad está cerca de la frontera; había habitantes bajo la categoría de refugiados, dos hechos que le impidieron recurrir a las encuestas o a los cuestionarios. Fue consciente de que se trataba de un acercamiento pionero tocante a una problemática definida por factores endógenos y exógenos. Hernández Castillo capitalizó en esta investigación la experiencia que había tenido al estudiar en ejidos de la zona las condiciones de vida de refugiados k'anjobales (Hernández, 1989: 129).

En el momento del estudio la población del ejido Cuauhtémoc era en su mayoría refugiada guatemalteca. La investigadora observó la continuidad lingüística existente más allá de la demarcación geográfica. Vio que se trataba de un área amplia con hablantes de chuj y k'anjobal, expresiones lingüísticas excluidas de los catálogos y las monografías financiados por el Estado mexicano: "Jacaltecos, mames, chujes y k'anjobales, han sido por mucho tiempo 'los olvidados de la frontera', es hasta hace pocos años que algunos investigadores empiezan a centrar su atención en estas comunidades" (Hernández, 1989: 113). Es una afirmación relevante. La investigadora puso en tensión las disposiciones del Estado mexicano y las definiciones que pudieran hacerse de los pobladores de esa amplia área de la frontera. Esa preocupación la orilló a elaborar la historia del ejido con el énfasis puesto en las denominaciones lingüísticas.

La región de los lagos de Montebello, donde se localizan los ejidos de Tziscao y Cuauhtémoc, se pobló a finales del siglo XIX. Hubo dos coincidencias: la expropiación de las tierras comunales en Guatemala y la necesidad del Estado mexicano de poblar la línea fronteriza, que aún se estaba trazando. La mexicanización de la frontera se incitó por medio de campañas coercitivas que se extendieron hasta entrado el siglo XX (Hernández, 1989: 139). La escuela fue un medio para lograrla. La investigadora apuntó que la formación del ejido Cuauhtémoc fue la manera en que algunos pobladores de Tziscao respondieron a las presiones gubernamentales y a la de un cacique llegado a la región.

La frontera como espacio de definición de una colectividad frente a las presiones externas: las del Estado mexicano y las del Estado guatemalteco. Uno en busca de la formación de la nación mexicana; el otro, con la persecución de la población indígena. Y esa colectividad, con sus diferencias internas; las observadas por la investigadora fueron las de tipo religioso, sobre todo. Estuvo entre sus preocupaciones poner el acento en la denominación lingüística como elemen- 
to vinculante, y la costumbre como aglutinadora de creencias: "En el caso de Cuauhtémoc, a pesar del aparente abandono de los rituales tradicionales y de la participación activa en otras formas de religiosidad, el respeto hacia la costumbre persiste y en muchos casos se mantiene una participación paralela en ambos ámbitos religiosos" (Hernández, 1989: 155). La autora entrevistó a personas mayores, a autoridades civiles y religiosas. Ubicó grupos según la denominación religiosa profesada por los habitantes de la localidad. Dispuso que "la costumbre" debía ser el punto de partida del estudio del cambio religioso.

La antropóloga constató la utilización que el presbiterianismo hizo de las lenguas del lugar para allegarse adeptos. Las lenguas ignoradas por el Estado mexicano fueron el vehículo mediante el cual presbiterianos de Cuauhtémoc accedieron a la lectura de la Biblia: "los refugiados presbiterianos vinieron a revitalizar la utilización del idioma indígena al interior del servicio religioso y a recuperar la tradición musical" (Hernández, 1989: 204). Este mecanismo fue un aglutinador de las poblaciones refugiada y mexicana. Los refugiados fueron los más inclinados a volver a "los valores culturales tradicionales" para salvaguardarse de las presiones externas. La investigadora ubicó el lugar de la Teología de la Liberación como propulsora de proyectos que dejaban de lado lo que podía identificarse como el ritual tradicional. Asimismo, corroboró que las denominaciones religiosas fueron impulsadas por personas en espacios y tiempos concretos: "lo religioso es un espacio abierto y sujeto a constantes transformaciones" (Hernández, 1989: 207).

Rosalva Aída Hernádez Castillo utilizó un oxímoron: "Recordando la historia olvidada: Tziscao y Cuauhtémoc, dos comunidades fronterizas" en el título del texto que escribió con base en conversaciones que sostuvo con habitantes de Cuauhtémoc; los datos sobre el ejido de Tziscao los recopiló Jorge Luis Cruz Burguete. Se hizo una historia ilustrada. Los dibujos provocan que se evoque a habitantes de los Altos de Chiapas. Es una narración hecha por periodos, sin que sea posible identificar los argumentos en los que se sostuvo ese fallo. Es posible identificar el idioma y la cultura como inquietudes de la investigadora: "Por sobre las prohibiciones del pasado el idioma y la cultura sobreviven en estas dos comunidades fronterizas bajo nuevas formas y con nuevos retos" (Hernández, 1989: 266). Este documento pasó a formar parte del libro Religión y sociedad en el sureste de México, volumen II, el cual contiene los resultados de investigación escritos por Jorge Luis Cruz Burguete, sobre Tziscao, y Rosalva Aída Hernández Castillo, sobre Cuauhtémoc. 
El cambio religioso, en la investigación coordinada por Fábregas con la protección académica del CIESAS-Sureste, fue la variable que condujo el quehacer de los investigadores. La frontera fue percibida en función de las dos localidades seleccionadas: Tziscao y Cuauhtémoc. La primera de estas se concibió dentro de la política mexicana con la que se delimitó el país. Se detectó que durante la primera mitad del siglo XX habitantes de uno y otro lado de la frontera mantenían contacto de índole familiar, sobre todo. Las persecuciones en Guatemala cambiaron la intensidad de esa movilidad. En ambos ejidos fueron asentados guatemaltecos bajo la categoría de "refugiados". Con ellos, hicieron su aparición en la zona las denominaciones religiosas no católicas. La Iglesia católica se instaló en la frontera, como protectora de los refugiados. Los investigadores, en sus afirmaciones, en la definición de sus respectivos textos, mostraron sus inclinaciones políticas, su forma de concebir lo que podría ser ese universo al que se habían enfrentado. Las estancias en las localidades estuvieron circunscritas al año que duró el proyecto. Cada uno hizo uso de los recursos que tuvo a su alcance: la teoría, en un caso; la exploración anterior de la zona, en el otro.

\section{Fronteras étnicas}

La población refugiada atrajo la atención sobre la frontera sur. Personas de Guatemala asentadas en México; ahí, la definición de una circunstancia: refugiadas, dentro de los ejidos o en terrenos aledaños. En este universo, me interesa destacar cómo esta condición de la frontera sur provocó que Edith F. Kauffer Michel (2005) se preguntara por el tipo de relación que los refugiados mantenían con el resto de los habitantes de esta zona del país. Centró su análisis entre la frontera política y la frontera étnica. Puso en práctica el concepto de frontera en dos procesos: el del refugio y el de la integración. Diez años de trabajo de campo con los refugiados le permitieron hacer este análisis publicado en la revista Frontera Norte. La publicación del artículo se dio 20 años después de la aparición del libro La formación histórica de la frontera sur coordinado por Andrés Fábregas Puig.

Kauffer partió de las dos definiciones de frontera que han sido útiles en las ciencias sociales: la frontera como límite y la frontera como frente. Ubicó su procedencia; la formulación inicial fue hecha por Frederick Jackson Turner en 1893. Ella tomó para sí que la frontera como límite era la que definía la situación que 
se vivió en la frontera sur al momento en que llegaron los refugiados a la zona. El Estado mexicano buscó reforzar el límite internacional. Acontecimientos posteriores originados en México y allende sus fronteras provocaron que se intensificara esta determinación:

Este proceso de conformación de la frontera sur, entendido como el reforzamiento de la existencia del límite internacional, ha sido posteriormente alimentado por otros acontecimientos muy particulares tales como el levantamiento armado zapatista de 1994, la migración indocumentada centroamericana en tránsito hacia Estados Unidos y, más recientemente, la 'amenaza terrorista' derivada del 11 de septiembre de 2001 (Kauffer, 2005: 9).

La frontera como frente fue visible con la llegada de los refugiados, quienes se internaron hacia México en busca de tierras dónde vivir; se ubicaron en espacios despoblados, sobre todo, para formar campamentos. Fue más notoria la frontera frente cuando el gobierno mexicano decidió ubicar a poblaciones refugiadas en los estados de Campeche y Quintana Roo; la frontera agrícola fue abierta por estas poblaciones.

Kauffer buscó una definición dilatada de la frontera política; para llegar a ésta, recurrió a la que de frontera elaboró Michel Foucher: "una estructura espacial de forma lineal con una función de discontinuidad geográfica y demarcación, de ubicación en tres registros: real, simbólico e imaginario" (Kauffer, 2005: 12). La discontinuidad puede situarse "entre soberanías, historias, sociedades, economías y Estados" (Kauffer, 2005: 12). Los registros enmarcados en lo simbólico y en el imaginario le serán de utilidad a esta autora para comprender la frontera política en el momento del refugio.

Hernández Castillo (1989) y Cruz Burguete (1989) formularon que la frontera política dio tranquilidad a los refugiados; fue un signo de supervivencia, indicó Kauffer (2005), quien vio también que esa línea que había representado un espacio de protección ofreció a los refugiados el intersticio para pensar en el retorno, impulsado por líderes políticos, quienes imaginaban un futuro en la tierra de origen. Ese futuro no llegó. Y algunos retornados volvieron a México: "Este 'juego de ir y venir' nos indica que la frontera y su 'más allá' alimentan una movilidad regional y que el cruce de la línea de división internacional se convierte en una manera de resolver ciertos problemas inmediatos, políticos o económicos" (Kauffer, 2005: 15). La población que había llegado con el refugio siguió transitando por la frontera, hacia uno y otro lado. 
Edith Kauffer estuvo interesada en analizar las identidades que surgieron con la presencia en México de población guatemalteca que buscaba refugio. La primera que se reconoció fue la de "refugiado"; la segunda, mediante trabajo político, la "guatemalteca". Para comprenderlas, Kauffer se basó en la definición de frontera escrita por Foucher (Kauffer, 2005: 16). Trabajó en localidades situadas en el estado de Chiapas. Las zonas zapatistas y un proceso electoral la orillaron a dividir el trabajo de campo en dos regiones geográficas; ambas con población guatemalteca. En la primera ubicó los municipios de "La Trinitaria (parte baja), Frontera Comalapa, Bellavista y Amatenango de la Frontera" (Kauffer, 2005: 18); "Marqués de Comillas, Maravilla Tenejapa, Las Margaritas, La Independencia y la Trinitaria (parte alta), en la segunda" (Kauffer, 2005: 18). Recopiló la información mediante encuestas y entrevistas.

Una vez descritas las zonas, delimitó su posición con respecto a las fronteras étnicas; se auxilió de los planteamientos de Barth, Poutignat y Streiff-Fénart:

Por etnicidad no se entiende la pertenencia a un grupo de origen indígena o nativo como lo planteaba la antropología de los años cincuenta con un enfoque etnocentrista, sino una categorización que resulta de la interacción social, la cual se caracteriza por elementos de diferenciación que tienen un significado para los protagonistas (Kauffer, 2005: 20-21).

De la identificación de "refugiado" y "guatemalteco" se pasó a la dificultad para nombrar a quienes habían decidido quedarse en México. Las indefiniciones se daban en el ámbito gubernamental, en el de las organizaciones de apoyo y en el de la diaria convivencia. Kauffer encontró cómo fue variando la condición de quienes aparecieron como refugiados, sin tierra. Muchos lograron comprar terrenos y buscar mejores condiciones de vida. Otros vieron la migración como un hecho. Conforme se fue dando el proceso de integración, la circunstancia étnica fue cediendo terreno. Al momento del refugio, la etnicidad se presentó como un elemento aglutinador. Con la integración, las decisiones fueron individuales; quedó lejos cualquier intencionalidad que abogara por la colectividad (Kauffer, 2005: 35).

El concepto de frontera fue colocado en los ámbitos de la etnicidad. Se le observó también en las maneras en que los refugiados se ubicaron en la línea territorial; y de ahí, a cómo buscaron formas de asegurar su subsistencia. Edith Kauffer (2005) prestó atención a las relaciones entre unos, mexicanos, y otros, refugiados; entre unos, integrados, y otros, exrefugiados. 


\section{Frontera: cultura y política}

A veinte años de la publicación de su libro sobre la formación histórica de la frontera sur, Andrés Fábregas Puig (2005) hizo una formulación del concepto de frontera. Retomó ideas que había trabajado con anterioridad (Fábregas 1997; 2003). Situó la reflexión entre sus preocupaciones por observar la continuidad cultural que existe hacia el sur de México y la importancia de la región para el estudio de las fronteras. Advirtió que podía utilizarse el concepto de varias formas y con significados diversos. De la proposición de Frederick Jackson Turner (1893) al esbozo de Alejandro Grimson (2000). En el primero, la frontera como frente, como espacio vacío para ser conquistado; en el segundo, la frontera como sitio de "encuentro de relatos"; como parte de esas dimensiones de análisis, la frontera en relación con el Estado nacional (Fábregas, 2005: 21).

Fábregas descubrió que Turner, sin advertirlo, introdujo una cuestión relevante al analizar la frontera: las ecologías culturales eran alteradas al ser colonizadas. Este punto fue seguido por autores como Julian Steward, Karl Wittfogel, Karl Marx y Owen Lattimore, cuyas reflexiones estuvieron situadas en el Oriente. Esas discusiones del siglo XIX y las producidas durante el siglo XX le permitieron a Fábregas afirmar "que las fronteras son territorios en los que la interacción de agentes internos y externos configura los acontecimientos locales. [...] Las fronteras son espacios y ámbitos de imbricación social y cultural, de formación de sociedades y culturas particulares, y de generación del cambio social" (Fábregas, 2005: 27).

Las acciones humanas han definido los límites entre los Estados nacionales. Intereses determinados han guiado esas actuaciones. La relación entre la sociedad y el medio se produce en ámbitos regionales, a los que la frontera se encuentra vinculada (Fábregas, 2005: 29). De ese modo, al poner el énfasis en la territorialidad, acentuó que "la antropología debe insistir en que las regiones y sus fronteras no son espacios dados naturales, sino el resultado de elaboraciones históricas de procesos sociales concretos" (Fábregas, 2005: 31). La región debe ser parte del problema de investigación, debe estar en la metodología. Este precepto le ayudó a observar que es inexistente una sola forma de comprender la región. Él se avino a la definición dada por Pedro Tomé; propuso cuatro niveles de análisis imbricados. Las redes sociales en función de los ambientes naturales; las personas en esas redes con sus respectivas actividades, con las que se transforma y crea los ecosistemas culturales; los factores externos que influyen 
en las redes; y la interdependencia de los tres ámbitos que modelan la región (Fábregas, 2005: 32).

La frontera como parte de una región fue una propuesta que Fábregas hizo en 1985. El objeto de estudio era la frontera sur. La formulación del 2005 se alejó de la especificidad. Situó los ejemplos en el ámbito de América Latina. Instaló la discusión en las definiciones nacionales. La frontera norte de México tiene como núcleo básico la identidad cultural; la frontera sur está definida por la dimensión política de los Estados nacionales.

Frente a la Frontera Norte, México se asume como una comunidad de cultura, con sus límites reconocidos, de cara a otra comunidad de cultura que es Estados Unidos. Frente a la Frontera Sur, México se reconoce como una comunidad política en contraste con otras comunidades políticas, cuyos límites se contienen en las territorialidades abarcadas por los Estados nacionales (Fábregas, 2005: 35).

Asumió la existencia de culturas similares en los territorios que están en la frontera sur. Siguió pensando en la continuidad cultural que marca la especificidad del sur de México y Centroamérica. Hacia el norte del país, una comunidad de cultura; hacia el sur, una comunidad política. Así resumió el contraste histórico de la sociedad mexicana (Fábregas, 2005: 36). La comunidad de cultura ha sido estudiada en el norte de México; la comunidad política, en la frontera sur, con el énfasis en las repercusiones económicas de los tratados comerciales. Ubicó el estudio de la frontera en el ámbito de los Estados nacionales de América Latina; vio esta enfrentada a los Estados Unidos: "En este contexto, todo México es frontera, como comunidad de cultura y como comunidad política" (Fábregas, 2005: 39). Esas comunidades darían lugar a "configuraciones regionales" que requerirían ser examinadas. Su comprensión de esta gran frontera se nutrió de ejemplos extraídos de Europa (Fábregas, 2005: 40).

Identificó tres ejes temáticos relevantes para la comprensión de la frontera: a) los contextos generados por el colonialismo reciente, en un ambiente globalizado, donde sean considerados los procesos locales; b) las fronteras internas en relación con la territorialidad nacional y las disputas regionales por el poder; c) las articulaciones entre frontera, región y nación (Fábregas 2005:41). Estos ejes deben ser examinados sin obviar el principio propuesto por Frederick Jackson Turner: la frontera como tierra vacía, en la que el otro enfrentado carece de existencia. Percibió que la de Turner era una noción vigente, al examinar algunos ejemplos contemporáneos. Esa expansión en pos de nuevos territorios no hace homogéneos los espacios conquistados: "el mundo actual en su cotidianidad se 
mueve en ámbitos localizados, delimitados, conectándose las interacciones sociales con espacios determinados" (Fábregas, 2005: 42). La antropología, expuso Fábregas, "posee una larga experiencia en el análisis de estos microuniversos y de sus fronteras" (Fábregas, 2005: 43).

Vio también la utilidad de la propuesta de Owen Lattimore: la frontera como producto del quehacer humano, en ecologías culturales. Reforzó así la importancia del trabajo de campo en el conocimiento de las fronteras, que no son ni únicas ni inamovibles. Su propuesta metodológica contempló que "el análisis de las fronteras debe ser comparativo, etnográfico y dotado de medios históricos" (Fábregas, 2005: 45). Del dato empírico a la reflexión teórica. Al ser alterables las ecologías culturales, había que considerar los vínculos entre la ecología cultural y la historia; la situación específica del microcosmos debía ser analizada; el concepto de "contexto transformativo" era una herramienta para hacer estos enlaces. El contexto de Centroamérica se estaba transformando a partir de las intensas migraciones hacia el Norte. Estaban surgiendo las pandillas juveniles; avanzaban hacia la frontera sur de México. Las investigaciones sobre estos nuevos contextos debían rebasar la cuantificación de los migrantes. El estudio de la frontera con sólido trabajo de campo se hacía indispensable (Fábregas, 2005: 48).

Andrés Fábregas Puig ha tenido una perspectiva amplia sobre la frontera sur de México. La ha recorrido. Su reflexión antropológica se ha alimentado de sus estancias en la entidad chiapaneca. Le ha sido necesario ponderar la etapa en que estuvo al frente del proyecto sobre la frontera sur, en los años ochenta del siglo XX (Fábregas, 2011; 2015); se abrieron temáticas antes inexploradas sobre todo en Chiapas. Se enriqueció el quehacer antropológico. Ha insistido en la importancia del trabajo de campo. Él ha explorado con dilección los Altos de Jalisco. Ha analizado las fronteras de la tierra nómada. Su mirada hacia el Sur ha sido intermitente. Cuando ha estado en posibilidades de aumentar sus indagaciones, cierta circunstancia política lo ha apartado físicamente del límite meridional de México. Su propuesta metodológica sobre cómo deben ser estudiados los contextos regionales del sur del país está compendiada en el artículo de 2005, "El concepto de frontera: una formulación", publicado en el libro Fronteras desbordadas.

La concepción de la frontera como frente está asociada a procesos de colonización. El territorio conquistado es visto como tierra de nadie. Se le domina. No hay alguien enfrente que sea identificable. Esa fue la forma en que Frederick Jackson Turner la expuso, según se ha citado líneas arriba. En el artículo "Frontera, pro- 
cesos migratorios y autonómicos en la conformación territorial mam: cambios y perspectivas" (Piña y Fábregas, 2015), Joaquín Peña Piña y Andrés Fábregas Puig ensayaron la explicación de Turner. Observaron procesos de identidad surgidos mediante la reinvención de lo indígena en la localidad Pavencul, del municipio de Tapachula, Chiapas. Consideraron de utilidad teórica referirse a los límites establecidos en esa localidad y a la, así llamada por ellos, expansión de la cultura mam en los Estados Unidos de América:

Así, como Frederick Jackson Turner explica al hablar de la expansión de la frontera americana, del mismo modo los migrantes mames en Estados Unidos, a través de su trabajo y el establecimiento de relaciones sociales y económicas, expanden su influencia para trazar nuevas fronteras en otro territorio. Los mecanismos colectivos de apoyo y solidaridad se fortalecen en el territorio de llegada se van conformando redes sociales similares a las de la comunidad de origen (Peña y Fábregas, 2015: 74).

Una localidad en movimiento que expandió sus fronteras a partir de proyectos colectivos. Vista de esa forma, la noción de frontera fue utilizada por los autores del texto sin reparar en que omitieron un componente: el de la dominación, según la propuesta hecha por Frederick Jackson Turner. Tuvieron el propósito de decir cómo una colectividad puede propagarse por medio de estrategias de migración y autonomía. Y para hacer el análisis recurrieron a las dos proposiciones de frontera sobre las que Fábregas (2005) ha escrito, como límite y como frente de expansión, y a las temporadas de campo que tuvo Peña (2004) en la región alta de Tapachula. Dos autores fueron su guía: Owen Lattimore y Frederick Jackson Turner. Y se tomaron la libertad de pensar la frontera solo como frente de expansión. Prescindieron del sentido de dominio que ese axioma conlleva. Sugirieron que era necesario revisar el concepto de frontera como límite, en virtud de cómo las localidades se mueven más allá de sus territorios. Concluyeron que el concepto de frontera como frente cobraba un mayor sentido a partir de "los procesos de globalización que buscan flexibilizar y evitar el establecimiento de fronteras y que paradójicamente reafirman lo local e influyen en el control del territorio" (Peña y Fábregas, 2015: 78).

\section{Borde}

Hace más de 30 años, la frontera sur de México surgió como campo de investigación de la antropología. En este texto me propuse dilucidar cómo había sido 
definido el concepto de frontera por quienes en ese tiempo buscaron comprender los acontecimientos suscitados a partir de los años ochenta del siglo XX en el límite internacional Chiapas-Guatemala, sobre todo. Encontré destacable la formulación que tuvo como soporte teórico el planteamiento de Frederick Jackson Turner, que no fue consignado en los textos iniciales. Andrés Fábregas tuvo a bien pensar en las regiones de la frontera sur. Ha insistido en esa idea. Su quehacer antropológico lo ha orillado a puntualizar la importancia del trabajo de campo (Fábregas, 2011: 64, 82); labor necesaria si se pone en entredicho la idea que expresaron Peña y Fábregas, citada líneas arriba, sobre cómo la globalización podría impedir que sean instauradas fronteras.

Siguen siendo vigentes estos postulados: trabajo de campo y teoría. Estado, región y frontera. Las regiones y las fronteras dentro de procesos de globalización. Estas premisas son ubicables como preguntas de investigación. Las fui extrayendo de las ideas expuestas líneas arriba; el borde como una condición actual.

Me inclino por el estudio de la frontera. Aún es terreno inexplorado, a pesar de que se le nombre a partir de cierta visión de una sola vez y para siempre (véase Villafuerte, 2017). Con el acercamiento expuesto en este texto, quise leer la forma en que se empezó a hacer antropología en la frontera Chipas-Guatemala. Leí a los antecesores (apuntes de una conversación), con la idea de situarlos. Aún hay misterios por descifrar.

\section{Fuentes de consulta}

Caballero Trejo, Nidya Fernanda, 2014, El refugio de la memoria. La Comisión Mexicana de Límites entre México y Guatemala, 1878-1899, México, Secretaría de Relaciones Exteriores.

Castillo, Manuel Ángel, Mónica Toussaint y Mario Vázquez Olivera, 2006, Espacios diversos, historia en común. México, Guatemala y Belice: la construcción de una frontera, México, Secretaría de Relaciones Exteriores.

Cruz Burguete, Jorge Luis, 1989, "Tziscao", en Andrés Fábregas, Carlos Guzmán Böckler, Jorge Luis Cruz Burguete y Rosalva Aída Hernández Castillo, Religión y sociedad en el sureste de México, v. II, México, Centro de Investigaciones y Estudios Superiores en Antropología Social, cuadernos de la Casa Chata 162, pp. 35-122.

Fábregas, Andrés, 1985, "Introducción", en Fábregas, Andrés, Juan Pohlenz, Mariano Báez y Gabriel Macías, La conformación histórica de la frontera sur, México, Centro de Investigaciones y Estudios Superiores en Antropología Social-Sureste/Secretaría de Educación Pública, cuadernos de la Casa Chata 124, pp. 3-22. 
Fábregas Puig, Andrés, 2005, "El concepto de frontera: una formulación", en Alain Basail Rodríguez (coordinador), Fronteras des-bordadas. Ensayos sobre la frontera sur de México, México, Casa Juan Pablos/Universidad de Ciencias y Artes de Chiapas, pp. 21-51.

Fábregas Puig, Andrés, 2011, Configuraciones regionales mexicanas. Un planteamiento antropológico, tomo II, México, Universidad Intercultural de Chiapas.

Hernández Castillo, Rosalva Aída, 1989, "Del tzolkin a la Atalaya: los cambios en la religiosidad en una comunidad chuj-k'anjobal de Chiapas", en Andrés Fábregas, Carlos Guzmán Böckler, Jorge Luis Cruz Burguete y Rosalva Aída Hernández Castillo, Religión y sociedad en el sureste de México, v. II, México, Centro de Investigaciones y Estudios Superiores en Antropología Social, cuadernos de la Casa Chata 162, pp. 123-224.

Hernández Castillo, Rosalva Aída, 2001, La otra frontera. Identidades múltiples en el Chiapas poscolonial, México, Centro de Investigaciones y Estudios Superiores en Antropología Social/Miguel Ángel Porrúa.

Hernández Castillo, Rosalva Aída, 2012, Sur profundo. Identidades indígenas en la frontera Chiapas-Guatemala, Centro de Investigaciones y Estudios Superiores en Antropología Social/Comisión para el Desarrollo de los Pueblos Indígenas, México.

Hernández Palacios, Luis y Juan Manuel Sandoval (comps.), 1989, El redescubrimiento de la frontera sur, México, Universidad Autónoma de Zacatecas/Universidad Autónoma Metropolitana.

Kauffer Michel, Edith F., 2005, "De la frontera política a las fronteras étnicas: refugiados guatemaltecos en México", Frontera Norte, 17(34), pp. 7-36.

Peña Piña, Joaquín, 2004, "Migración laboral de las mujeres y estrategias de reproducción social en una comunidad indígena mam de la Sierra Madre de Chiapas, México", tesis para obtener el grado de doctor en Ciencias en Ecología y Desarrollo Sustentable, San Cristóbal de Las Casas, Chiapas, El Colegio de la Frontera Sur.

Peña Piña, Joaquín y Andrés Fábregas Puig, 2015, "Frontera, procesos migratorios y autonómicos en la conformación territorial mam: cambios y perspectivas", LiminaR. Estudios sociales y humanísticos, XIII(2), pp. 62-83.

Pohlenz, Juan, 1985, "La formación de la frontera entre México y Guatemala. El caso de Nuevo Huixtán en la selva chiapaneca", en Andrés Fábregas, Juan Pohlenz, Mariano Báez y Gabriel Macías, 1985, La conformación histórica de la frontera sur, México, Centro de Investigaciones y Estudios Superiores en Antropología Social-Sureste/ Secretaría de Educación Pública, cuadernos de la Casa Chata 124, pp. 23-130.

Ponce Jiménez, Patricia, 1985, Palabra viva del Soconusco, Centro de Estudios Superiores en Antropología Social/Secretaría de Educación Pública, México.

Villafuerte Solís, Daniel, 2017, Tiempo de fronteras. Una visión geopolítica de la frontera sur de México, Juan Pablos Editor/Universidad de Ciencias y Artes de Chiapas/ Centro de Estudios Superiores de México y Centroamérica, México. 